\title{
A NEW SIMILARITY MEASURE BETWEEN HMMS
}

\author{
Yih-Ru Wang \\ Department of Communication Engineering, National Chiao Tung University, Hsinchu \\ yrwang@mail.nctu.edu. tw
}

\begin{abstract}
In this paper, a new similarity measure between HMM models which extended the well-known Kullback-Leibler distance was proposed. The Kullback-Leibler distance was defined as the mean of log-likelihood ratio (LLR) in a hypotheses test and the Kullback-Leibler distance was frequently used as a similarity measure for HMM models. Here, the standard deviation of LLR between HMM models was deviated first. Besides, the ratio of mean and standard variation of LLR was used as a new similarity measure between HMM models. Experiments were done in a Mandarin speech database, TCC-300, in order to check the effectiveness of the proposed similarity measure. The accuracy of the standard deviation of LLR estimated from the syllable HMM models was checked by comparison with the standard deviation of LLR of top-10 candidates found from HMM decoder. And, the confusion sets of 411 syllables were also found by using both the $\mathrm{KL}$ distance and the proposed similarity measure. Comparing to the top- 10 confusion models, $94.9 \%$ and $95.3 \%$ inclusion rates can be achieved by using KL distance and the proposed similarity measure of HMM models.
\end{abstract}

Index Terms - similarity measure, Kullback-Leibler distance, Hidden Markov Model

\section{INTRODUCTION}

The HMM was the most frequently used acoustic decoder for speech recognition system. In the HMM speech recognition system, after a set of HMM models were trained from the training database, the performance of the models was usually found by decoding a large test speech database using the given models. The loglikelihoods for phone/word hypotheses were used for decision making in HMM speech recognition system. To model the loglikelihood ratio, it was also called similarity or distance, between HMM models directly from the models was an interested research topic since the HMM model was proposed [1].

If the log-likelihood ratio between HMM models can be properly modeled, it can be used in lots of applications. In the previous researches, lots of works were done in order to find the log-likelihood ratio (LLR), or similarity, between two HMM models [2-5]. The Kullback-Leibler distance was usually been used to describe the similarity between states of two HMM models. The Kullback-Leibler distance was original to describe the crossentropy of a detector. In the ML recognizer, the Kullback-Leibler distance was also the mean of log-likelihood ratio between two hypotheses.

The similarity measure using Kullback-Leibler distance was used in lots of applications. The similarity/distance measure of

This work was supported by the National Science Council, Taiwan, under the project with contract NSC 96-2221-E-009 -041.
HMM has been used in evaluation of the HMM re-estimation [1]; redefinition of acoustics unit [6]; multilingual phoneme mapping [7]; vocabulary selection [8], pronunciation variation analysis [9] and error rate estimation of HMM models [10].

But the log-likelihood ratio between two HMM models was a random variable, and the Kullback-Leibler distance is in fact the mean of log-likelihood ratio. The larger mean of the log-likelihood ratio between two models did not render a smaller classification error. If some high order statistics of LLR was known, then a better similarity measure can be found to determine the confusion set of recognizer.

In this paper, the estimated standard deviation of the loglikelihood ratio between two HMM models was first deviated. And, the normalized LLR mean, i.e., mean normalized by standard deviation, between two HMM models was found. And, the confusion mono-syllables sets were found from the normalized LLR mean between the HMM models. Finally, the TCC-300 Mandarin speech database was used to validate the effectiveness of the proposed similarity measure.

\section{THE SIMILARITY MEASURE BETWEEN HMM MODELS}

In HMM decoder, the log-likelihood ratio (LLR) for a given test data, $\mathbf{O}$, can be expressed as

$$
\log \left(p\left(\mathbf{O} \mid \Lambda_{i}\right)\right)-\log \left(p\left(\mathbf{O} \mid \Lambda_{j}\right)\right) ; \forall j \neq i,
$$

where $\Lambda_{i}$ is the HMM model of a word/phone hypotheses and the $\Lambda_{j}$ is a competitive model.

We note that this log-likelihood ratio is itself a random variable with probability distribution function

$$
p_{\Lambda_{i}}\left(\log \left(p\left(\Lambda_{i}\right)\right)-\log \left(p\left(\Lambda_{j}\right)\right)\right) ; \forall j \neq i \text {. }
$$

From this above distribution function, not only the first order statistics can be used to define the similarity of two HMM models. The high order statistics, such as variance, can also used to find a better similarity measure. In the following subsections, the variance of LLR between two probability distributions will first be derived and then extended to two HMM models.

\subsection{Mean and variance of LLR between two random variables}

For two random variables with distributions $p_{1}(\mathbf{X})$ and $p_{2}(\mathbf{X})$, the mean of log-likelihood ratio between them can be expressed as

$E_{p_{1}(\mathbf{X})}\left(\log \left(p_{1}(\mathbf{X})\right)-\log \left(p_{2}(\mathbf{X})\right)\right)=\int p_{1}(\mathbf{X}) \log \frac{p_{1}(\mathbf{X})}{p_{2}(\mathbf{X})} d \mathbf{X}$
$=\int p_{1}(\mathbf{X})\left(\log \left(p_{1}(\mathbf{X})\right)-\log \left(p_{2}(\mathbf{X})\right)\right) d \mathbf{X}$ 
The above expectation of the log-likelihood ratio is the famous Kullback-Leibler distance (KL-distance) $[11,12]$ or also called the quadratic discriminate function in statistics. If these two distributions were both Gaussian, i.e., $p_{1}(\mathbf{X})=N\left(\boldsymbol{\mu}_{1}, \boldsymbol{\Sigma}_{1}\right)$ and $p_{2}(\mathbf{X})=N\left(\boldsymbol{\mu}_{2}, \boldsymbol{\Sigma}_{2}\right)$, then the KL-distance can be represented as

$E_{p_{1}(\mathbf{X})}\left(\log \left(p_{1}(\mathbf{X})\right)-\log \left(p_{2}(\mathbf{X})\right)\right)$

$=\frac{1}{2}\left(\log \frac{\left|\boldsymbol{\Sigma}_{2}\right|}{\left|\boldsymbol{\Sigma}_{1}\right|}+\operatorname{tr}\left(\boldsymbol{\Sigma}_{2}^{-1} \boldsymbol{\Sigma}_{1}-\mathbf{I}\right)+\left(\boldsymbol{\mu}_{2}-\boldsymbol{\mu}_{1}\right)^{T} \boldsymbol{\Sigma}_{2}^{-1}\left(\boldsymbol{\mu}_{2}-\boldsymbol{\mu}_{1}\right)\right)$.

In this paper, the variance of $\left(\log \left(p_{1}(\mathbf{X})\right)-\log \left(p_{2}(\mathbf{X})\right)\right)$, will be first deviated. By complicated matrix computations, we can find the variance of log-likelihood ratio between two Gaussian distributions.

First, by definition the variance of LLR between two Gaussian distributions can be expressed as,

$$
\begin{aligned}
V_{p_{1}(\mathbf{X})}\left(\log \left(p_{1}(\mathbf{X})\right)-\log \left(p_{2}(\mathbf{X})\right)\right) \\
=\frac{1}{4} V_{p_{1}(\mathbf{X})}\left(\left(\mathbf{X}-\boldsymbol{\mu}_{2}\right)^{T} \boldsymbol{\Sigma}_{2}^{-1}\left(\mathbf{X}-\boldsymbol{\mu}_{2}\right)-\left(\mathbf{X}-\boldsymbol{\mu}_{1}\right)^{T} \boldsymbol{\Sigma}_{1}^{-1}\left(\mathbf{X}-\boldsymbol{\mu}_{1}\right)\right) \\
=\frac{1}{4} E_{p_{1}(\mathbf{X})}\left(\left\{\left(\mathbf{X}-\boldsymbol{\mu}_{2}\right)^{T} \boldsymbol{\Sigma}_{2}^{-1}\left(\mathbf{X}-\boldsymbol{\mu}_{2}\right)-\left(\mathbf{X}-\boldsymbol{\mu}_{1}\right)^{T} \boldsymbol{\Sigma}_{1}^{-1}\left(\mathbf{X}-\boldsymbol{\mu}_{1}\right)\right\}^{2}\right) \\
-\frac{1}{4}\left(\operatorname{tr}\left[\left(\boldsymbol{\mu}_{1}-\boldsymbol{\mu}_{2}\right)^{T} \boldsymbol{\Sigma}_{2}^{-1}\left(\boldsymbol{\mu}_{1}-\boldsymbol{\mu}_{2}\right)\right]+\operatorname{tr}\left[\boldsymbol{\Sigma}_{2}^{-1} \boldsymbol{\Sigma}_{1}\right]-\operatorname{tr}[\mathbf{I}]\right)^{2}
\end{aligned}
$$

Then, the product terms in Eq. (3) can be simplified as,

$$
\begin{aligned}
& \left(\mathbf{X}-\boldsymbol{\mu}_{i}\right)^{T} \boldsymbol{\Sigma}_{i}^{-1}\left(\mathbf{X}-\boldsymbol{\mu}_{i}\right)\left(\mathbf{X}-\boldsymbol{\mu}_{j}\right)^{T} \boldsymbol{\Sigma}_{j}^{-1}\left(\mathbf{X}-\boldsymbol{\mu}_{j}\right) \\
& =\left(\left(\mathbf{X}-\boldsymbol{\mu}_{1}\right)^{T} \boldsymbol{\Sigma}_{i}^{-1}\left(\mathbf{X}-\boldsymbol{\mu}_{1}\right)\left(\mathbf{X}-\boldsymbol{\mu}_{1}\right)^{T} \boldsymbol{\Sigma}_{j}^{-1}\left(\mathbf{X}-\boldsymbol{\mu}_{1}\right)\right) \\
& \quad+\left(\left(\boldsymbol{\mu}_{1}-\boldsymbol{\mu}_{i}\right)^{T} \boldsymbol{\Sigma}_{i}^{-1}\left(\boldsymbol{\mu}_{1}-\boldsymbol{\mu}_{i}\right)\left(\boldsymbol{\mu}_{1}-\boldsymbol{\mu}_{j}\right)^{T} \boldsymbol{\Sigma}_{j}^{-1}\left(\boldsymbol{\mu}_{1}-\boldsymbol{\mu}_{j}\right)\right) \\
& \quad+4\left(\boldsymbol{\mu}_{1}-\boldsymbol{\mu}_{i}\right)^{T} \boldsymbol{\Sigma}_{i}^{-1}\left(\mathbf{X}-\boldsymbol{\mu}_{1}\right)\left(\boldsymbol{\mu}_{1}-\boldsymbol{\mu}_{j}\right)^{T} \boldsymbol{\Sigma}_{j}^{-1}\left(\mathbf{X}-\boldsymbol{\mu}_{1}\right) . \\
& \quad+\left(\boldsymbol{\mu}_{1}-\boldsymbol{\mu}_{i}\right)^{T} \boldsymbol{\Sigma}_{i}^{-1}\left(\boldsymbol{\mu}_{1}-\boldsymbol{\mu}_{i}\right)\left(\mathbf{X}-\boldsymbol{\mu}_{1}\right)^{T} \boldsymbol{\Sigma}_{j}^{-1}\left(\mathbf{X}-\boldsymbol{\mu}_{1}\right) \\
& +\left(\boldsymbol{\mu}_{1}-\boldsymbol{\mu}_{j}\right)^{T} \boldsymbol{\Sigma}_{j}^{-1}\left(\boldsymbol{\mu}_{1}-\boldsymbol{\mu}_{j}\right)\left(\mathbf{X}-\boldsymbol{\mu}_{1}\right)^{T} \boldsymbol{\Sigma}_{i}^{-1}\left(\mathbf{X}-\boldsymbol{\mu}_{1}\right) \\
& +Q\left(\left(\mathbf{X}-\boldsymbol{\mu}_{1}\right)^{n} ; n=1,3\right)
\end{aligned}
$$

And, $E\left[Q\left(\left(\mathbf{X}-\boldsymbol{\mu}_{1}\right)^{n} ; n=1,3\right)\right]$ will be zero for Gaussian random variables. Let $\boldsymbol{\Sigma}_{1}^{-1}=\mathbf{L} \mathbf{U}=\mathbf{L} \mathbf{L}^{T}$. Then,

$\mathbf{U}\left(\mathbf{X}-\boldsymbol{\mu}_{1}\right)=\mathbf{Y}, \quad\left(\mathbf{X}-\boldsymbol{\mu}_{1}\right)^{T} \mathbf{L}=\mathbf{Y}^{T}$ where $\mathbf{Y} \sim N(0, \mathbf{I})$.

The expectation of the first term in Eq. (4) is

$$
\begin{aligned}
& E_{p_{1}(\mathbf{X})}\left(\operatorname{tr}\left(\left(\mathbf{X}-\boldsymbol{\mu}_{1}\right)^{T} \boldsymbol{\Sigma}_{i}^{-1}\left(\mathbf{X}-\boldsymbol{\mu}_{1}\right)\left(\mathbf{X}-\boldsymbol{\mu}_{1}\right)^{T} \boldsymbol{\Sigma}_{j}^{-1}\left(\mathbf{X}-\boldsymbol{\mu}_{1}\right)\right)\right) \\
& =E_{p_{1}(\mathbf{X})}\left(\operatorname{tr}\left(\mathbf{Y}^{T} \mathbf{L}^{-1} \boldsymbol{\Sigma}_{i}^{-1} \mathbf{U}^{-1} \mathbf{Y} \mathbf{Y}^{T} \mathbf{L}^{-1} \boldsymbol{\Sigma}_{j}^{-1} \mathbf{U}^{-1} \mathbf{Y}\right)\right) \\
& =\operatorname{tr}\left(\mathbf{L}^{-1} \boldsymbol{\Sigma}_{i}^{-1} \mathbf{U}^{-1} \mathbf{L}^{-1} \boldsymbol{\Sigma}_{j}^{-1} \mathbf{U}^{-1} \cdot E_{p_{1}(\mathbf{X})}\left(\mathbf{Y} \mathbf{Y}^{T} \mathbf{Y} \mathbf{Y}^{T}\right)\right) \\
& =\operatorname{tr}\left(\mathbf{L}^{-1} \boldsymbol{\Sigma}_{i}^{-1} \mathbf{U}^{-1} \mathbf{L}^{-1} \boldsymbol{\Sigma}_{j}^{-1} \mathbf{U}^{-1} \cdot(2 \mathbf{I}+\operatorname{tr}(\mathbf{I}) \cdot \mathbf{I})\right) \\
& =(d+2) \operatorname{tr}\left(\mathbf{L}^{-1} \boldsymbol{\Sigma}_{i}^{-1} \mathbf{U}^{-1} \mathbf{L}^{-1} \boldsymbol{\Sigma}_{j}^{-1} \mathbf{U}^{-1}\right) \\
& =(d+2) \operatorname{tr}\left(\boldsymbol{\Sigma}_{i}^{-1} \mathbf{U}^{-1} \mathbf{L}^{-1} \boldsymbol{\Sigma}_{j}^{-1} \mathbf{U}^{-1} \mathbf{L}^{-1}\right)=(d+2) \operatorname{tr}\left(\boldsymbol{\Sigma}_{1}^{2} \boldsymbol{\Sigma}_{i}^{-1} \boldsymbol{\Sigma}_{j}^{-1}\right)
\end{aligned}
$$

where $\left(\mathbf{L}^{-1} \boldsymbol{\Sigma}_{i}^{-1} \mathbf{U}^{-1}\right)^{T}=\left(\mathbf{U}^{-1}\right)^{T} \boldsymbol{\Sigma}_{i}^{-1}\left(\mathbf{L}^{-1}\right)^{T}=\mathbf{L}^{-1} \boldsymbol{\Sigma}_{i}^{-1} \mathbf{U}^{-1}$ is symmetric. Thus, we can change the order of matrix multiplications inside the trace operation. The expectation of Eq. (4) becomes

$$
\begin{aligned}
& E_{p_{1}(\mathbf{X})}\left(\left(\mathbf{X}-\boldsymbol{\mu}_{i}\right)^{T} \boldsymbol{\Sigma}_{i}^{-1}\left(\mathbf{X}-\boldsymbol{\mu}_{i}\right)\left(\mathbf{X}-\boldsymbol{\mu}_{j}\right)^{T} \boldsymbol{\Sigma}_{j}^{-1}\left(\mathbf{X}-\boldsymbol{\mu}_{j}\right)\right) \\
& =(d+2) \operatorname{tr}\left(\boldsymbol{\Sigma}_{1}^{2} \boldsymbol{\Sigma}_{i}^{-1} \boldsymbol{\Sigma}_{j}^{-1}\right)+4\left(\boldsymbol{\mu}_{1}-\boldsymbol{\mu}_{i}\right)^{T} \boldsymbol{\Sigma}_{i}^{-1} \boldsymbol{\Sigma}_{1} \boldsymbol{\Sigma}_{j}^{-1}\left(\boldsymbol{\mu}_{1}-\boldsymbol{\mu}_{j}\right) \\
& +\left(\boldsymbol{\mu}_{1}-\boldsymbol{\mu}_{i}\right)^{T} \boldsymbol{\Sigma}_{i}^{-1}\left(\boldsymbol{\mu}_{1}-\boldsymbol{\mu}_{i}\right)\left(\boldsymbol{\mu}_{1}-\boldsymbol{\mu}_{j}\right)^{T} \boldsymbol{\Sigma}_{j}^{-1}\left(\boldsymbol{\mu}_{1}-\boldsymbol{\mu}_{j}\right) \\
& +\left(\boldsymbol{\mu}_{1}-\boldsymbol{\mu}_{i}\right)^{T} \boldsymbol{\Sigma}_{i}^{-1}\left(\boldsymbol{\mu}_{1}-\boldsymbol{\mu}_{i}\right) E_{p_{1}(\mathbf{X})}\left(\left(\mathbf{X}-\boldsymbol{\mu}_{1}\right)^{T} \boldsymbol{\Sigma}_{j}^{-1}\left(\mathbf{X}-\boldsymbol{\mu}_{1}\right)\right) \\
& +\left(\boldsymbol{\mu}_{1}-\boldsymbol{\mu}_{j}\right)^{T} \boldsymbol{\Sigma}_{j}^{-1}\left(\boldsymbol{\mu}_{1}-\boldsymbol{\mu}_{j}\right) E_{p_{1}(\mathbf{X})}\left(\left(\mathbf{X}-\boldsymbol{\mu}_{1}\right)^{T} \boldsymbol{\Sigma}_{i}^{-1}\left(\mathbf{X}-\boldsymbol{\mu}_{1}\right)\right)
\end{aligned}
$$

And, since

$$
E_{p_{1}(\mathbf{X})}\left(\left(\mathbf{X}-\boldsymbol{\mu}_{1}\right)^{T} \boldsymbol{\Sigma}_{j}^{-1}\left(\mathbf{X}-\boldsymbol{\mu}_{1}\right)\right)=\left\{\begin{array}{cc}
d & \text {,if } j=1 \\
\operatorname{tr}\left(\boldsymbol{\Sigma}_{1} \boldsymbol{\Sigma}_{j}^{-1}\right) & , \text { if } j \neq 1
\end{array}\right.
$$

Then, after simplification, we have

$$
\begin{aligned}
V_{p_{1}(\mathbf{X})}\left(\left(\mathbf{X}-\boldsymbol{\mu}_{2}\right)^{T} \boldsymbol{\Sigma}_{2}^{-1}\left(\mathbf{X}-\boldsymbol{\mu}_{2}\right)-\left(\mathbf{X}-\boldsymbol{\mu}_{1}\right)^{T} \boldsymbol{\Sigma}_{1}^{-1}\left(\mathbf{X}-\boldsymbol{\mu}_{1}\right)\right) \\
=E_{p_{1}(\mathbf{X})}\left(\left(\left(\mathbf{X}-\boldsymbol{\mu}_{2}\right)^{T} \boldsymbol{\Sigma}_{2}^{-1}\left(\mathbf{X}-\boldsymbol{\mu}_{2}\right)-\left(\mathbf{X}-\boldsymbol{\mu}_{1}\right)^{T} \boldsymbol{\Sigma}_{1}^{-1}\left(\mathbf{X}-\boldsymbol{\mu}_{1}\right)\right)^{2}\right) \\
-\left(\operatorname{tr}\left[\left(\boldsymbol{\mu}_{1}-\boldsymbol{\mu}_{2}\right)^{T} \boldsymbol{\Sigma}_{2}^{-1}\left(\boldsymbol{\mu}_{1}-\boldsymbol{\mu}_{2}\right)\right]+\operatorname{tr}\left[\boldsymbol{\Sigma}_{2}^{-1} \boldsymbol{\Sigma}_{1}\right]-\operatorname{tr}[\mathbf{I}]\right)^{2}, \\
=(d+2)\left(\operatorname{tr}\left[\boldsymbol{\Sigma}_{1}^{2} \boldsymbol{\Sigma}_{2}^{-2}\right]-2 \operatorname{tr}\left[\boldsymbol{\Sigma}_{1} \boldsymbol{\Sigma}_{2}^{-1}\right]+d\right) \\
-\left(\operatorname{tr}\left[\boldsymbol{\Sigma}_{2}^{-1} \boldsymbol{\Sigma}_{1}-\mathbf{I}\right]\right)^{2}+4\left(\boldsymbol{\mu}_{1}-\boldsymbol{\mu}_{2}\right)^{T} \boldsymbol{\Sigma}_{2}^{-1} \boldsymbol{\Sigma}_{1} \boldsymbol{\Sigma}_{2}^{-1}\left(\boldsymbol{\mu}_{1}-\boldsymbol{\mu}_{2}\right)
\end{aligned}
$$

Finally, the variance of log-likelihood ratio of two Gaussiandistributed random variables can be expressed as,

$$
\begin{aligned}
& V_{p_{1}(\mathbf{X})}\left(\log \left(p_{1}(\mathbf{X})\right)-\log \left(p_{2}(\mathbf{X})\right)\right) \\
& =\frac{1}{4}\left((d+2) \operatorname{tr}\left[\left(\boldsymbol{\Sigma}_{2}^{-1} \boldsymbol{\Sigma}_{1}-\mathbf{I}\right)^{2}\right]-\left(\operatorname{tr}\left[\boldsymbol{\Sigma}_{2}^{-1} \boldsymbol{\Sigma}_{1}-\mathbf{I}\right]\right)^{2}\right) \\
& \quad+\left(\boldsymbol{\mu}_{1}-\boldsymbol{\mu}_{2}\right)^{T} \boldsymbol{\Sigma}_{2}^{-1} \boldsymbol{\Sigma}_{1} \boldsymbol{\Sigma}_{2}^{-1}\left(\boldsymbol{\mu}_{1}-\boldsymbol{\mu}_{2}\right)
\end{aligned}
$$

\subsection{Mean and variance of LLR between two HMM models}

After finding the mean and variance of log-likelihood ratio between two Gaussian distributions, we will extend it to the mean and variance of log-likelihood ratio between two HMM models. When the observation probability in each state of HMM model was Gaussian distributed, the mean and variance of the log-likelihood ratio of a given states in two HMM models can be obtained. In the HMM model, the mixture Gaussian observation probability was usually used, and denoted as

$$
b(\mathbf{X})=\sum_{i} c_{i} \cdot N\left(\boldsymbol{\mu}_{i}, \boldsymbol{\Sigma}_{i}\right)
$$

where $\boldsymbol{\Sigma}_{i}$ are usually diagonal covariance matrices.

First, a single Gaussian distribution, $p(\mathbf{X})=N(\boldsymbol{\mu}, \boldsymbol{\Sigma})$, was used to approximate the observation probability of each HMM state. The mean vector, $\boldsymbol{\mu}$, and covariance matrix, $\boldsymbol{\Sigma}$, of the observation probability can be found from the following formulas 
$\boldsymbol{\mu}=\sum_{i} c_{i} \cdot \boldsymbol{\mu}_{i}$

$[\Sigma]_{m n}=\sum_{i} c_{i} \cdot\left(\left[\Sigma_{i}\right]_{m n}+\left[\boldsymbol{\mu}_{i}\right]_{m}\left[\boldsymbol{\mu}_{i}\right]_{n}\right)-[\boldsymbol{\mu}]_{m}[\boldsymbol{\mu}]_{n}$,

where $\Sigma$ is a full covariance matrix and $[\Sigma]_{m n}$ is the element of covariance matrix.

According to the maximum likelihood criterion of HMM decoding, the minimum log-likelihood ratio between two HMM models $\Lambda_{i}$ and $\Lambda_{j}$, where $\Lambda=(A, B, \Pi)$, can be expressed in terms of KL-distance between states in two HMM models, i.e.,

$$
\begin{aligned}
& E_{\Lambda_{i}}\left(\log \left(p\left(\Lambda_{i}\right)\right)-\log \left(p\left(\Lambda_{j}\right)\right)\right) \\
& =\underset{(\mathbf{s} \mathbf{1} \mathbf{2} \mathbf{2})}{\operatorname{MIN}} \sum_{s 1} d_{s 1} \cdot\left\{E_{\Lambda_{i}}\left(\log \left(b_{s 1}\left(X, \Lambda_{i}\right)\right)-\log \left(b_{s 2}\left(X, \Lambda_{j}\right)\right)\right)\right\} \quad \forall j \neq i
\end{aligned}
$$

(8)

where $d_{i}$ is the average state duration of state $i$, and which can be found from the state transition probability, $A$, in HMM model, i.e., $d_{i}=1 /\left(1-a_{i i}\right)$. And the minima of Eq. (8) were found from the all possible state mapping from $(s 1=0, s 2=0)$ to $(s 1=\boldsymbol{S i}, s 2=\boldsymbol{S j})$, where $\boldsymbol{S} \boldsymbol{i}$ is the number of state for $\Lambda_{i}$. And, the log-likelihood ratio due to transition probabilities in HMM model can be easily added into Eq. (8).

Now, a dynamic time warping can be used to find the optimal state mapping, $(\hat{s} 1, \hat{s} 2)$. And the optimal state sequence mapping can be found from

$$
(\hat{s} 1, \hat{s} 2)=\operatorname{Arg} \underset{(\mathbf{s 1}, \mathbf{s} 2)}{\operatorname{MIN}} \sum_{s 1} d_{s 1} \cdot\left\{E_{\Lambda_{i}}\left(\begin{array}{l}
\log \left(b_{\hat{s} 1}\left(X, \Lambda_{i}\right)\right)- \\
\log \left(b_{\hat{s} 2}\left(X, \Lambda_{j}\right)\right)
\end{array}\right)\right\} ; \forall j \neq i
$$

If HMM modeling, the observation probabilities for different states in HMM and different input speech frames were assumed uncorrelated. Thus, the covariance of the log-likelihood ratio can be expressed as

$$
\begin{aligned}
& V_{\Lambda_{i}}\left(\log \left(p\left(\Lambda_{i}\right)\right)-\log \left(p\left(\Lambda_{j}\right)\right), \log \left(p\left(\Lambda_{i}\right)\right)-\log \left(p\left(\Lambda_{j}\right)\right)\right) \\
& =\sum_{(\hat{s} 1, \hat{s} 2)} d_{s 1} \cdot\left\{V_{\Lambda_{i}}\left(\begin{array}{c}
\log \left(b_{\hat{s} 1}\left(X, \Lambda_{i}\right)\right)-\log \left(b_{\hat{s} 2}\left(X, \Lambda_{j}\right)\right), \\
\log \left(b_{\hat{s} 1}\left(X, \Lambda_{i}\right)\right)-\log \left(b_{\hat{s} 2}\left(X, \Lambda_{j}\right)\right)
\end{array}\right)\right\} \quad \forall j \neq i
\end{aligned}
$$

where the state mapping $(\hat{s} 1, \hat{s} 2)$ was the optimal state mapping found in Eq. (9).

Finally, the normalized mean, mean normalized by the standard deviation, was used as the similarity measure between two HMM models.

\section{EXPERIMENT RESULTS AND DISCUSSIONS}

The TCC-300 Mandarin speech database was used in the following experiments to examine the effectiveness of the model of loglikelihood ratio between HMM models. The input speech signal was sampled in $16 \mathrm{kHz}$ with 16-bit linear PCM format. A 38 recognition features including $12 \mathrm{MFCCs}$ with their first and second derivatives, and delta and delta-delta log-energies. The analysis frame width was $30 \mathrm{~ms}$ with $10 \mathrm{~ms}$ frame shift. A window length of 5 frames was used in the calculations of derivatives. The speech data of 135 male and 135 female speakers in TCC-300 were used to train the 8-state HMMs for 411 Mandarin mono-syllables.
The total number of syllables was 301,337 . Beside the 301,337 training syllables mentioned above, 31,295 test syllables recorded by the others 15 female and 15 male speakers were also used to find the effectiveness of proposed similarity measure.

The speech data, totally 331,835 syllables, were first segmented into syllables using force-alignment. The mean of log-likelihood ratio (LLR) of the top-10 candidates were found from the HMM decoder and LLR mean estimated from HMM models using Eqs. (2), (7) and (8), were shown in Fig. 1, and the correlation coefficient was 0.87. As shown in Fig. 1, the Kullback-Leibler distances were good estimation of the LLR means thus they can be successfully used in the applications mentioned in Section 1.

The standard deviation of log-likelihood ratio between HMM models using HMM decoder and estimated from HMM models using Eqs. (5), (7) and (10), were shown in Fig. 2. And, the proposed similarity measure, i.e., mean divided by standard deviation of log-likelihood ratio, between HMM models found from HMM decoder and estimated from HMM models were shown in Fig. 3, and the correlation coefficient was 0.52. In Fig. 3, beside some outliers, the proposed similarity measure were well estimated. We can see that similarity measure of log-likelihood ratio between mono-syllable [si] and [shi] estimated from HMM models was much larger than the ratio found from HMM decoding. It was due the freqently happened mis-pronunciation in Mandarin. Lots of speakers will pronuce the $[\mathrm{shi}]$ as $[\mathrm{si}],[\mathrm{cu}]$ as $[\mathrm{chu}]$, [zong] as [zhong], ..., etc. And some outliers in Fig. 3, such as [lia] and [ne], were due to the numbers of training data were too little.

Then, the top-n confusion sets of Mandarin syllables were found from the HMM models by using serveral kinds of similarity measures. In the experiment, the confusion model sets of a target HMM was determined by using the following similarity measures (1) KL distance, or LLR mean of HMM models, (2) the proposed similarity measure, i.e., the estimated normalized mean of LLR from HMM models directly without decoding any speech data, and (3) normalized mean of LLR found from HMM decoder, Then, the most similar models for each 411 target model were chosen by using above similarity measures. The inclusion rates for target and the top-n confusion models were shown in Table 1. The proposed similarity measure will be better than KL distance and a little worse than real normailzed LLR found from HMM decoder. In comparison with the top-10 confusion models, $94.9 \%$ and $95.3 \%$ inclusion rates can be achieved by using KL distance and the proposed similarity measure of HMM models. Thus, the effective confusion sets of HMM recognizer can be found from the models directly without any speech data decoding needed.

Table. 1. The inclusion rates of the top-n confusion models determined from three different similarity measures.

\begin{tabular}{|c|c|c|c|}
\hline $\begin{array}{c}\text { Similarity } \\
\text { measures }\end{array}$ & $(1)$ & $(2)$ & $(3)$ \\
\hline Correct model & $91.3 \%$ & $91.3 \%$ & $91.3 \%$ \\
\hline $\begin{array}{c}\text { Correct/top-1 } \\
\text { confusion model }\end{array}$ & $92.5 \%$ & $92.6 \%$ & $93.6 \%$ \\
\hline $\begin{array}{c}\text { Correct/top-3 } \\
\text { confusion models }\end{array}$ & $93.3 \%$ & $93.7 \%$ & $94.7 \%$ \\
\hline $\begin{array}{c}\text { Correct/top-5 } \\
\text { confusion model }\end{array}$ & $94.2 \%$ & $94.5 \%$ & $95.2 \%$ \\
\hline $\begin{array}{c}\text { Correct/top-10 } \\
\text { confusion models }\end{array}$ & $94.9 \%$ & $95.3 \%$ & $96.0 \%$ \\
\hline
\end{tabular}




\section{CONCLUSIONS}

In this paper, the variance of the log-likelihood ratio between two HMM models was deviated. And, using the mean and standard deviation of the LLR between two HMM models, a new similarity measure was proposed. And, using the proposed similarity measure, the confusion syllables sets for 411 Mandarin syllables was found and the effectiveness of them was checked.

\section{REFERENCES}

[1] B. H. Juang and L. R. Rabiner, " A probabilistic distance measure for hidden Markov models, ", AT\&T Technical Journal. Vol. 64, No. 2, pp. 391-408, 1985.

[2] Minh N. Do, " Fast approximation of Kullback-Leibler distance for dependence trees and hidden Markov models, ", IEEE Signal Processing Letters, Vol. 10, No. 4, pp. 115- 118 , Apr. 2003.

[3] M. Falkhausen, H. Reininger, D. Wolf, " Calculation of distance measures between hidden Markov models, “, in Proc Eurospeech Madrid, pp. 1287-1490, 1995.

[4] M. Vihola, M. Harju, P. Salmela, J. Suontausta and J. Savela, " Two dissimilarity measures for HMMs and their

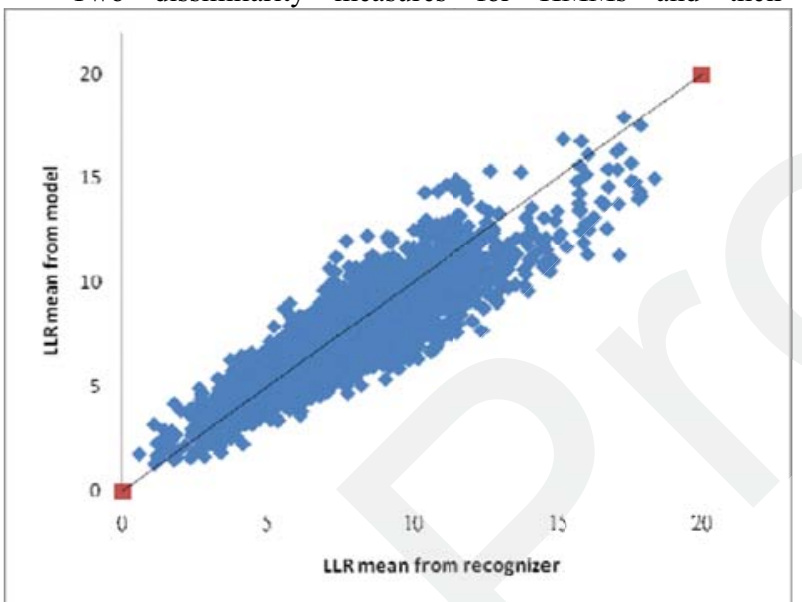

Fig. 1. The mean of log-likelihood ratio between HMM models using HMM decoder and model distance. (The loglikelihood ratio was normalized w.r.t. the number of frames) applications in phoneme model clustering, “, ICASSP-2002.

[5] J. Silva, S. Narayanan, " A Statistical Discrimination Measure for Hidden Markov Models based on Divergence, ", Interspeech 2004

[6] R. Singh, B. Raj., R. Stern, " Structured redefinition of sound units by merging and splitting for improved speech recognition, “ in ICSLP, 2000.

[7] Kohler J., " Multi-lingual phoneme recognition exploiting acoustic-phonetic similarities of sounds, “, in ICSLP 1996.

[8] P. Geutner, M. Finke, and A. Waibel, "Selection Criteria for hypothesis driven lexical adaptation, “, in ICASSP, 1999.

[9] M. Y. Tsai, and L. S. Lee, " Pronunciation Variations based on acoustic phonemic distance measures with application examples of Mandarin Chinese, ", in ASRU 2003.

[10] C. S. Jiand, H. C. Wang, C.H. Lee, " A Study on Modelbased Error-Rate Estimation for Automatic Speech Recognition, “, IEEE trans. On SA, pp. 581-589, Vol. 11, No. 6, NOV. 2003.

[11] Kullback, S., and Leibler, R. A., " On information and sufficiency, ", Annals of Mathematical Statistics 22, pp. 7986, 1951.

[12] Cover, T. M. and Thomas, J. A., 1991. Elements of Information Theory, New York: John Wiley and Sons.

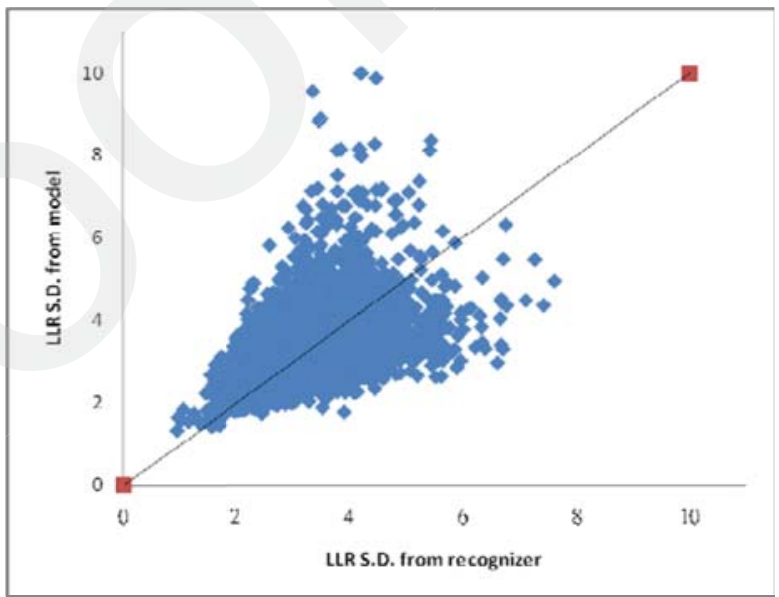

Fig. 2. The standard deviation of log-likelihood ratio between HMM models using HMM decoder and model distance.

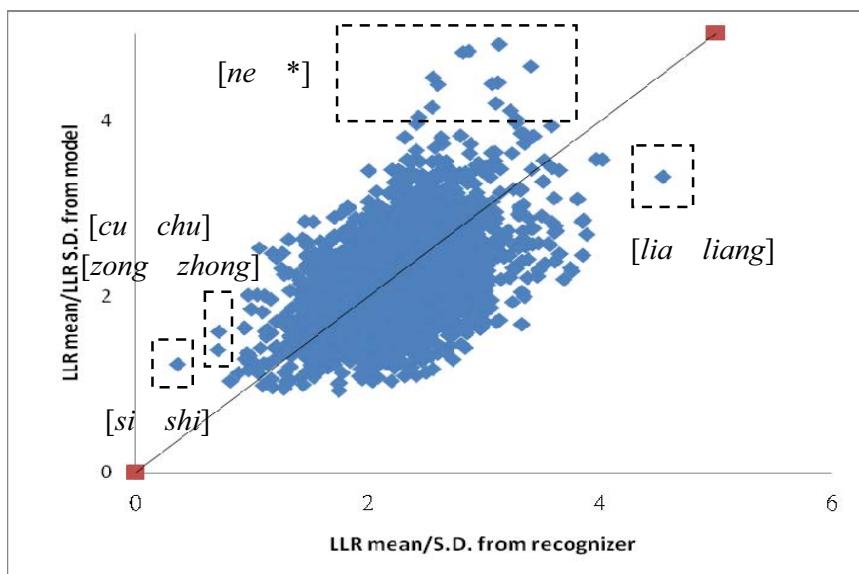

Fig. 3. The normalized mean (mean normalized by standard deviation) of log-likelihood ratio between HMM models using HMM decoder and model distance. 